\title{
Alleviation of Abdominal Pain due to Irinotecan-Induced Cholinergic Syndrome Using Loperamide: A Case Report
}

\author{
Kazuki Uchiyama ${ }^{a}$ Yoshitaka Saito $^{a}$ Yoh Takekuma $^{a}$ Satoshi Yuki ${ }^{b}$ \\ Mitsuru Sugawara ${ }^{a, c}$ \\ ${ }^{a}$ Department of Pharmacy, Hokkaido University Hospital, Sapporo, Japan; ${ }^{b}$ Department \\ of Gastroenterology and Hepatology, Hokkaido University Hospital, Sapporo, Japan; \\ 'Laboratory of Pharmacokinetics, Faculty of Pharmaceutical Sciences, Hokkaido University, \\ Sapporo, Japan
}

\section{Keywords}

Loperamide $\cdot$ Irinotecan $\cdot$ Abdominal pain $\cdot$ Cholinergic syndrome

\begin{abstract}
Irinotecan hydrochloride (irinotecan) is a chemotherapeutic agent used in the treatment of solid tumors. In addition to severe neutropenia and delayed diarrhea, irinotecan causes cholinergic syndrome, characterized by abdominal pain and acute diarrhea. The latter symptoms are frequently observed during and after irinotecan treatment. Here, we have discussed the case of a patient who completely recovered from abdominal pain following the administration of loperamide hydrochloride (loperamide) at a dose of $2 \mathrm{mg}$, before infusing irinotecan. In contrast, anticholinergic drugs were not as effective in alleviating symptoms. A 28-year-old man with stage IV rectal cancer with peritoneal metastasis was prescribed with fluorouracil, irinotecan, and levofolinate calcium (FOLFIRI), in addition to cetuximab. Anticholinergic drugs, such as scopolamine butylbromide (scopolamine) or atropine sulfate (atropine), were administered to treat abdominal pain that was considered as irinotecan-induced cholinergic syndrome, but monotherapy was not effective. Thereafter, oral loperamide $(2 \mathrm{mg})$ with atropine $(0.25 \mathrm{mg})$ was prescribed before irinotecan infusion. Consequently, the patient did not experience any abdominal pain during and after irinotecan treatment. Loperamide is an opioid receptor agonist and decreases the activity of the myenteric plexus of the intestinal wall. It also inhibits the release of both acetylcholine and prostaglandins, resulting in decreased inhibition of peristaltic movement. We assumed that its mechanism solely or in combination contributed to symptom relief. We hypothesized that the synergistic anticholinergic interaction between loperamide and atropine resulted in marked suppression of irinotecan-induced cholinergic syndrome compared to
\end{abstract}


loperamide alone. Thus, loperamide may improve abdominal pain attributed to irinotecaninduced cholinergic syndrome.

\section{Introduction}

Irinotecan hydrochloride (irinotecan) is a chemotherapeutic agent used in the treatment of several solid tumors, such as colorectal cancer and lung cancer [1]. Irinotecan is a prodrug that is extensively metabolized in the liver by carboxylesterase to produce the active metabolite 7-ethyl-10-hydroxycamptothecin (SN-38). It is then conjugated predominantly by liver UDP-glucuronosyltransferase (UGT) $1 \mathrm{~A} 1$ to form inactive SN-38 glucuronide (SN-38G) and is categorized as a topoisomerase I inhibitor [1]. In addition to severe neutropenia and delayed diarrhea, which are known to be dose-limiting toxicities of irinotecan, cholinergic syndrome is frequently observed during and after infusion of the drug. The symptoms of cholinergic syndrome include acute diarrhea, abdominal pain (peristaltic pain), nausea, sweating, nasal discharge, and lacrimation [2] and have been reported in up to $30-50 \%$ of Japanese patients [3]. These symptoms often reduce the patients' quality of life. Anticholinergic drugs such as scopolamine and atropine are effective against acute diarrhea caused by cholinergic syndrome [4].

We observed that the administration of $2 \mathrm{mg}$ loperamide hydrochloride (loperamide) plus $0.25 \mathrm{mg}$ atropine before infusing irinotecan markedly attenuated irinotecan-induced cholinergic abdominal pain. The anticholinergic drug alone was not effective, and our recommended treatment process has been reported herein.

\section{Case Report}

A 28-year-old man was diagnosed with stage IV rectal cancer with peritoneal metastasis (BRAF wild V600E, RAS wild type, non-MSI-H, UGT1A1*6 wild, and UGT1A1*28 wild). He was a nonsmoker and a social drinker with normal liver and renal function. He was subjected to chemotherapeutic treatment using FOLFIRI (fluorouracil $400 \mathrm{mg} / \mathrm{m}^{2}$ on day 1 and 2,400 mg/ $\mathrm{m}^{2}$ for $46 \mathrm{~h}$ from day 1 , irinotecan $150 \mathrm{mg} / \mathrm{m}^{2}$ on day 1 , and levofolinate calcium $200 \mathrm{mg} / \mathrm{m}^{2}$ on day 1, every 2 weeks) and cetuximab $\left(400 \mathrm{mg} / \mathrm{m}^{2}\right.$ for the first time, $250 \mathrm{mg} / \mathrm{m}^{2}$ in the subsequent injection, every week). Palonosetron $(0.75 \mathrm{mg}$ ) and dexamethasone $(9.9 \mathrm{mg})$ were intravenously administered on day 1 as antiemetic pre-medication for FOLFIRI administration, and chlorpheniramine maleate $(10 \mathrm{mg})$ was intravenously administered to prevent an infusion reaction with cetuximab. The concomitant drug prescribed was oral minocycline hydrochloride (50 mg twice daily) for skin toxicity prevention. The patient did not present with cancer-associated pain, including abdominal pain, and did not consume any analgesic agents during chemotherapy.

Details of the therapeutic process and supportive care are presented in Table 1. The patient experienced perspiration and abdominal pain during administration of irinotecan in the first course but did not experience diarrhea. Scopolamine $(20 \mathrm{mg}$ ) was intravenously administered because these symptoms were considered to be associated with cholinergic syndrome. Following treatment with scopolamine, the symptoms disappeared. During the second course, $20 \mathrm{mg}$ scopolamine was administered prior to irinotecan administration, but abdominal pain and articulatory disorder were reported during irinotecan infusion.

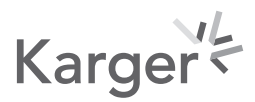


Table 1. Course details of symptoms and supportive care

\begin{tabular}{|c|c|}
\hline Course & Details of symptoms and supportive care \\
\hline $\mathrm{C} 1$ & $\begin{array}{l}\text { Perspiration and abdominal pain (peristaltic pain) were reported during administering } \\
\text { irinotecan. Scopolamine ( } 20 \mathrm{mg} \text { ) injection attenuated these symptoms }\end{array}$ \\
\hline $\mathrm{C} 2$ & $\begin{array}{l}\text { Scopolamine ( } 20 \mathrm{mg} \text { ) injection was preventively administered prior to irinotecan infusion, } \\
\text { but the effect was unsatisfactory. An additional injection of scopolamine ( } 20 \mathrm{mg} \text { ) successfully } \\
\text { resolved the symptoms }\end{array}$ \\
\hline $\mathrm{C} 3-\mathrm{C} 8$ & Scopolamine (20 mg) was intravenously administered before and after irinotecan infusion \\
\hline C9 & $\begin{array}{l}\text { Palpitations in addition to abdominal pain occurred during irinotecan infusion. Regimen was } \\
\text { changed from scopolamine }(20 \mathrm{mg}) \text { to atropine }(0.5 \mathrm{mg}) \text { injection before and after irinotecan } \\
\text { infusion. Abdominal pain was mitigated, but palpitations and respiratory discomfort were } \\
\text { reported }\end{array}$ \\
\hline C10-C14 & $\begin{array}{l}\text { Atropine injection was performed, ensuring a restricted total daily dose of } 1 \mathrm{mg} \text {, but all } \\
\text { regimens failed }\end{array}$ \\
\hline C15 & $\begin{array}{l}\text { Loperamide }(1 \mathrm{mg}) \text { was orally administered before irinotecan infusion in addition to } 0.25 \mathrm{mg} \\
\text { of atropine. Abdominal pain was mitigated, but the treatment was unsatisfactory }\end{array}$ \\
\hline $\mathrm{C} 20$ & $\begin{array}{l}\text { Oral loperamide }(2 \mathrm{mg}) \text { with atropine }(0.25 \mathrm{mg}) \text { was administered before irinotecan infusion. } \\
\text { Abdominal pain was completely resolved following treatment }\end{array}$ \\
\hline After C21 & No symptoms were reported \\
\hline
\end{tabular}

Accordingly, scopolamine (20 mg) was administered again after irinotecan infusion, and his symptoms improved. In the third course, scopolamine (20 mg) was intravenously administered before and after irinotecan infusion; however, the patient developed abdominal pain, and he claimed to experience palpitations during scopolamine administration. From the ninth course onward, we changed the pre-medication from scopolamine $(20 \mathrm{mg})$ to atropine $(0.5 \mathrm{mg})$ before and after irinotecan infusion. The patient's response seemed to support that atropine was superior to scopolamine in terms of attenuating symptoms of cholinergic syndrome. Nonetheless, the patient experienced palpitations and respiratory discomfort, which were more severe than those experienced when scopolamine was administered. Therefore, we injected atropine using approaches such as dose reduction from 0.5 to $0.25 \mathrm{mg}$ with frequent administration in the range of a total daily dose of $1 \mathrm{mg}$; however, success was not achieved. In the fifteenth course, we administered oral loperamide $(1 \mathrm{mg})$ with atropine $(0.25 \mathrm{mg})$ before irinotecan treatment. As a result, the symptoms partially improved. Thereafter, we increased the dosage of loperamide to $2 \mathrm{mg}$ while maintaining the atropine dose in the twentieth course. Following this regimen, the abdominal pain completely resolved, and administration of additional atropine was not necessary after irinotecan infusion.

\section{Discussion}

Two types of diarrhea reportedly occur following irinotecan treatment, namely acute and delayed diarrhea. Acute diarrhea occurs during or immediately after irinotecan infusion and is caused by increased cholinergic activity, which stimulates intestinal contractility and reduces the absorptive capacity of the mucosa [4]. The carbonyl group 
of irinotecan is considered to exert an inhibitory effect on acetylcholinesterase, and cholinergic syndrome may be caused by the stimulation of the muscarinic receptor due to excessive acetylcholine [5]. UGT1A1 is an important pharmacogenetic factor of irinotecan pharmacokinetics and affects the incidence and severity of delayed diarrhea [6]. Since the occurrence of acute diarrhea depends on irinotecan blood levels [7], no relationship exists between the UGT1A1 genotype and acute diarrhea caused by irinotecaninduced cholinergic syndrome. In general, anticholinergic agents such as scopolamine and atropine are used to treat this syndrome. In contrast, delayed diarrhea occurs approximately 8-10 days after irinotecan infusion and is probably caused by damage to the intestinal mucosa due to increased oxidative stress by biliary-secreted or intestinally deconjugated SN-38 [4]. Several guidelines recommend treatment of delayed diarrhea with loperamide or octreotide [8].

The predictive factors of irinotecan-induced cholinergic syndrome include irinotecan dose, gender (female), and combination chemotherapy $[1,5,9]$. Therefore, irinotecan dose reduction is one of the treatment strategies for preventing irinotecan-induced cholinergic syndrome; however, as easy dose reduction decreases the intensity of chemotherapy, alternative methods should be considered. In the previous studies, anticholinergic agents reportedly attenuated cholinergic syndrome [9]. Nevertheless, in the present study, the eventual addition of loperamide to an anticholinergic agent completely improved the patient's abdominal pain. The maximum plasma concentration of irinotecan is attained approximately $1 \mathrm{~h}$ after injection [10], and in the present study, the patient developed abdominal pain within $1 \mathrm{~h}$ of initiating irinotecan, which continued until day 2 . However, administration of $2 \mathrm{mg}$ loperamide suppressed not only immediate abdominal pain but also persistent pain. Since the half-life of loperamide is approximately $10 \mathrm{~h}$ [11], we assumed that loperamide contributed to the disappearance of abdominal symptoms considering its pharmacokinetics.

We formulated the following hypotheses on the mechanisms by which abdominal pain was alleviated following the administration of loperamide: (1) loperamide is an opioid receptor agonist that acts on the $\mu$-opioid receptors in the myenteric plexus of the large intestine [12]. Similar to opioids, loperamide decreases the activity of the myenteric plexus and the tone of the longitudinal and circular smooth muscles of the intestinal wall [12]. (2) The anticholinergic activity of loperamide helps relieve abdominal pain. A previous report has suggested that loperamide inhibits acetylcholine release, resulting in decreased peristaltic movement during circumferential distension of the intestinal wall [13]. Therefore, the mechanism of anticholinergic activity is different for loperamide, scopolamine, and atropine, with loperamide demonstrating higher competitive inhibition of the muscarinic acetylcholine receptor [9]. Furthermore, Awouters et al. [14] reported that loperamide exerted a higher effect on the intestine than anticholinergics, and opioids resulted in higher antidiarrheal activity in rats. (3) Inhibition of prostaglandin (PG) release by loperamide is also related to antidiarrheal activity [13]. Kase et al. [15] reported that irinotecan increased the release of $\mathrm{PGE}_{2}$, which is related to the onset of diarrhea, within $1 \mathrm{~h}$ of irinotecan injection in rats. Therefore, it may be suggested that loperamide inhibits biologically active substances that are related to the occurrence of diarrhea via multiple mechanisms. We assumed that the abovementioned mechanism of loperamide solely or in combination contributed to symptom relief in our patient.

Moreover, we consider that the synergistic anticholinergic interaction between loperamide and atropine facilitated further suppression of digestive disorders when compared to loperamide alone. In fact, since scopolamine or atropine dose escalation caused side effects such as palpitations and respiratory discomfort, we suggest that multidrug therapy is superior to monotherapy in certain cases.

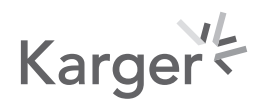




\section{Conclusion}

In the present study, we demonstrated the effectiveness of loperamide when anticholinergic agents such as scopolamine and atropine alone were ineffective in alleviating abdominal pain attributed to irinotecan-induced cholinergic syndrome. However, further studies on the use and effectiveness of loperamide in symptomatic relief are warranted.

\section{Statement of Ethics}

Written informed consent was obtained from the patient for the publication of this case report and any accompanying images.

\section{Conflict of Interest Statement}

K.U., Y.S., Y.T., S.Y., and M.S. have no conflicts of interest.

\section{Funding Sources}

This research received no specific grant from any funding agency in the public, commercial, or not-for-profit sectors.

\section{Author Contributions}

K.U. contributed to the design of the report, collected the data, and drafted the manuscript. Y.S., Y.T., S.Y., and M.S. revised the manuscript. All the authors read and approved the final version of the manuscript.

\section{References}

1 Tsuboya A, Fujita KI, Kubota Y, Ishida H, Taki-Takemoto I, Kamei D, et al. Coadministration of cytotoxic chemotherapeutic agents with irinotecan is a risk factor for irinotecan-induced cholinergic syndrome in Japanese patients with cancer. Int J Clin Oncol. 2019 Feb;24(2):222-30.

2 Rougier P, Bugat R, Douillard JY, Culine S, Suc E, Brunet P, et al. Phase II study of irinotecan in the treatment of advanced colorectal cancer in chemotherapy-naïve patients and patients pretreated with fluorouracilbased chemotherapy. J Clin Oncol. 1997 Jan;15(1):251-60.

3 Pitot HC, Goldberg RM, Reid JM, Sloan JA, Skaff PA, Erlichman C, et al. Phase I dose-finding and pharmacokinetic trial of irinotecan hydrochloride (CPT-11) using a once-every-three-week dosing schedule for patients with advanced solid tumor malignancy. Clin Cancer Res. 2000 Jun;6(6):2236-44.

4 de Man FM, Goey AKL, van Schaik RHN, Mathijssen RHJ, Bins S. Individualization of irinotecan treatment: a review of pharmacokinetics, pharmacodynamics, and pharmacogenetics. Clin Pharmacokinet. 2018 Oct; 57(10):1229-54.

5 Kanbayashi Y, Ishikawa T, Kanazawa M, Nakajima Y, Tabuchi Y, Kawano R, et al. Predictive factors for the development of irinotecan-related cholinergic syndrome using ordered logistic regression analysis. Med Oncol. 2018 Apr;35(6):82.

6 Hu ZY, Yu Q, Zhao YS. Dose-dependent association between UGT1A1*28 polymorphism and irinotecaninduced diarrhoea: a meta-analysis. Eur J Cancer. 2010 Jul;46(10):1856-65.

7 Hyatt JL, Tsurkan L, Morton CL, Yoon KJ, Harel M, Brumshtein B, et al. Inhibition of acetylcholinesterase by the anticancer prodrug CPT-11. Chem Biol Interact. 2005 Dec;157:247-52.

8 Benson AB 3rd, Ajani JA, Catalano RB, Engelking C, Kornblau SM, Martenson JA, et al. Recommended guidelines for the treatment of cancer treatment-induced diarrhea. J Clin Oncol. 2004 Jul;22(14):2918-26.

\section{Karger's}


9 Iihara H, Fujii H, Yoshimi C, Kobayashi R, Matsuhashi N, Takahashi T, et al. Prophylactic effect of scopolamine butylbromide, a competitive antagonist of muscarinic acetylcholine receptor, on irinotecan-related cholinergic syndrome. Cancer Chemother Pharmacol. 2019 Mar;83(3):393-8.

10 Chabot GG. Clinical pharmacokinetics of irinotecan. Clin Pharmacokinet. 1997 Oct;33(4):245-59.

11 Killinger JM, Weintraub HS, Fuller BL. Human pharmacokinetics and comparative bioavailability of loperamide hydrochloride. J Clin Pharmacol. 1979 Apr;19(4):211-8.

12 Hanauer SB. The role of loperamide in gastrointestinal disorders. Rev Gastroenterol Disord. 2008;8(1):15-20.

13 Yagasaki O, Suzuki H, Sohji Y. Effects of loperamide on acetylcholine and prostaglandin release from isolated guinea pig ileum. Jpn J Pharmacol. 1978 Dec;28(6):873-82.

14 Awouters F, Megens A, Verlinden M, Schuukes J, Niemegeers C, Janssen PA. Loperamide. Survey of studies on mechanism of its antidiarrheal activity. Dig Dis Sci. 1993 Jun;38(6):977-95.

15 Kase Y, Hayakawa T, Togashi Y, Kamataki T. Relevance of irinotecan hydrochloride-induced diarrhea to the level of prostaglandin E2 and water absorption of large intestine in rats. Jpn J Pharmacol. 1997 Dec;75(4): 399-405. 